\title{
El plan de nivelación académica Talentos, una respuesta institucional de educación para la paz*
}

\author{
Rafael Vergara Varela ${ }^{* *}$, María Eugenia Rodríguez Vásquez ${ }^{* * *}$
}

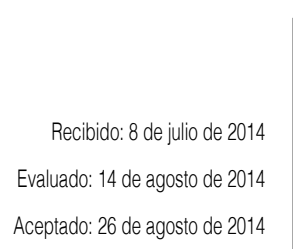

\section{RESUMEN}

En un contexto social desigual como en el que vive la ciudad de Santiago de Cali, donde gran parte de la juventud se desarrolla en medio de necesidades insatisfechas y altos índices de violencia, son necesarias acciones que permitan generar oportunidades y mejoras en los contextos donde se desarrollan los individuos.

Durante la alcaldía de Jorge Iván Ospina (2008-2010), en vista de la situación, se optó por la implementación de una serie de medidas que incidieran no solo en los individuos sino a la vez en sus entornos, surgiendo de esta manera entre sus políticas públicas el plan de nivelación académica Talentos, que tuvo como meta institucional mejorar la formación de los jóvenes, egresados de los planteles del área metropolitana; hecho que se vislumbró en la mejora en las pruebas de Estado Icfes y en el posterior ingreso de un porcentaje de la población tratamiento a la educación superior.

Palabras clave: política pública, juventud, educación para paz, capital humano, contexto social.

Las opiniones expresadas en este documento comprometen únicamente a sus autores y no a las entidades involucradas. Cómo citar este artículo: Vergara Varela, R. y Rodríguez Vásquez, M.E. (2014). El plan de nivelación académica Talentos, una respuesta institucional de educación para la paz. Hallazgos, 11(22), 403-417.

** Magíster en Políticas Públicas de la Universidad del Valle, Santiago de Cali, Colombia. Profesor del Instituto de Educación y Pedagogía de la Universidad del Valle, Santiago de Cali, Colombia. E-mail: rafael.vergara@correounivalle.edu.co.

*** Asistente de investigación. Estudiante del programa de Estudios Políticos y Resolución de Conflictos, séptimo semestre, Universidad del Valle, Santiago de Cali, Colombia. E-mail: maria.rodriguez.vasquez@correounivalle.edu.co 


\section{The Talentos academic plan, an institutional response of education for peace}

\section{Abstract}

In an unequal social context like the one of the city of Santiago de Cali, where a big part of Youth develops between unsatisfied needs and high levels of violence, necessary actions are needed to generate opportunities and improvements on contexts in which the individuals develop.

In view of this situation, During the term of mayor Jorge Ivan Ospina (2008 - 2010), it was implemented a series of measures having an impact not only on individuals but at the same time in their environments, thus emerging between his public policy the academic grading plan Talents, which had the institutional goal to improve the formation of young people, graduates of Schools in the metropolitan area, looming improved ICFES state tests and the subsequent entry to higher education of a percentage of the treatment population.

Keywords: Public policy, youth, education for peace, human capital, social context. 


\section{O plano de ensino de recuperação Talentos,}

\section{uma resposta institucional à educação para a paz}

\section{Resumo}

Num contexto social desigual como aquele no qual vive a cidade de Santiago de Cali, onde grande parte da juventude se desenrola no meio de necessidades insatisfeitas e altos níveis de violência são necessárias ações que permitem gerar oportunidades e melhoras nos contextos onde se desenvolvem os indivíduos.

Durante o período de prefeito Jorge Ivan Ospina (2008-2010), tendo em vista a situação, decidiu-se a implementação de uma série de medidas com impacto não só sobre os indivíduos, mas ao mesmo tempo em seus ambientes surgindo assim, entre as políticas públicas o plano de nivelamento acadêmico Talentos, que teve o objetivo institucional melhorar a formação dos jovens, formados nas escolas da região metropolitana; um fato que foi idealizado para melhorar os testes Estaduais ICFES e a subsequente entrada de uma porcentagem da população ao ensino superior.

Palavras-chave: políticas públicas, juventude, educação para a paz, o capital humano, contexto social. 


\section{Políticas públicas de educación para la Paz, Cali}

La ciudad de Santiago de Cali en los últimos 40 años ha estado creciendo de forma vertiginosa; de igual forma, ha sido la respuesta institucional a la sociedad; sin embargo, debido a múltiples conflictos urbanos, y al ser polo de concentración de flujos migratorios provenientes del sur occidente colombiano, ha desarrollado en la última década un aumento desmesurado en los niveles de pobreza. Para 2007, mediante el sistema de identificación de potenciales beneficiarios de programas sociales (Sisben) se registró e identifico que había 1.200.827 personas, de las cuales el 56,6\% del total de la población es residente en los estratos socioeconómicos uno, dos y tres. De dicha población registrada por este sistema, el 42,6\% se clasificó en el nivel dos de pobreza, que corresponde a personas que poseen al menos una necesidad básica insatisfecha (NBI); seguida por los niveles uno con un $34,3 \%$; que se refieren a población en extrema pobreza o con dos o más NBI; y en el nivel tres quedó clasificado el 22,9\% de los encuestados, los cuales no son pobres técnicamente (Alcaldía de Santiago de Cali, 2008, p. 6).

La población total de la ciudad para 2009 estaba conformada por 2.219.633 de habitantes, de los cuales el $18 \%$ se encontraba entre los 16 y 24 años de edad; para el año siguiente según cifras del Departamento Administrativo Nacional de Estadística (Dane) había 400.576 habitantes entre los 15 y 24 años de edad (García Viera, 2013), y eran definidos, según el Congreso de la Republica de Colombia mediante la Ley 375 de 1997, como jóvenes. Empero, diversos autores (Margulis, Urresti, Martin-Barbero y Valenzuela, 1998) plantean que el concepto de juventud no tiene definición como tal sino que este es el resultado de la sumatoria de características sociales, económicas, políticas, culturales y de género (García Viera, 2013, pp. 24-25).

La ciudad, debido a la crisis social en la cual ha estado inmersa, ha llevado a que gran cantidad de los jóvenes que en ella conviven se involucren en actos de violencia y vandalismo, vivan en condiciones de pobreza, marginaciones y conflictos de todo tipo; y de esta manera se presentó al hombre como el lobo del hombre y se originó una lucha por diversos intereses, dado que buscan satisfacer necesidades básicas y que su entorno no les permite desarrollarse integralmente debido a la falta de oportunidades y de acceso a la educación, siendo esta última fundamental para modificar sus vidas y el contexto donde conviven.

Ahora bien, la educación como conjunto de prácticas sociales es el principal medio e instrumento para solucionar las problemáticas que aquejan a la sociedad, tales como falta de oportunidades, problemas de convivencia, injusticias, participación y paz, entre otros; urgiendo, como plantea Zurbano (1999, p. 13), a una educación para la paz, esto significa la implementación de un modelo pedagógico que desarrolle actitudes y comportamientos coherentes con la justicia, el respeto, el diálogo y la resolución de conflictos. Claro está que dicha educación debe ser incentivada por parte del Estado, ya que con ello no solo genera un aumento en el capital humano de los ciudadanos, sino que a la vez incrementa el capital de la nación, hecho que impacta tanto en temáticas como en cultura democrática y formación ciudadana, y a la vez afecta la productividad individual y la del país.

Aunque al hablar de capital humano se hace referencia a un concepto relativamente 
moderno, este fue anunciado por Schultz (1968) como diversas formas que generan flujos adicionales de ingreso que inciden en el crecimiento económico, lo cual modificó salarios en términos absolutos y relativos. De esta manera, el acervo en calificaciones y conocimientos aumenta la productividad, por ello se necesitan inversiones constantes y a temprana edad en los individuos, para que así estos se conviertan en un factor de dicha productividad (Leyva y Cárdenas; 2002). Como plantean Cunha y Heckman (2007a, 2008b), la mitad de la desigualdad en el valor presente de los ingresos de la vida se debe a factores que se encuentran determinados a los 18 años de edad.

Como expresa Castrillón (2013), la distribución desigual da origen a diversos grupos de ingresos o estratos socioeconómicos, que ocasionan que quienes poseen bajos ingresos y por ende distintos tipos de medios (recursos, bienes, etc.) se enfrenten a limitaciones de todo tipo que restringen y condicionan sus capacidades para la acumulación de capital humano, siendo este último resultado del ahorro realizado para invertir en educación, con el fin de incidir en el valor de mercado del trabajo (Ray, 1998, citado por Castrillón, 2013, p. 1).

En cuanto a la educación, en especial la superior y con calidad, esta se encuentra en un contexto donde la gran mayoría de su oferta está privatizada, lo que hace difícil acceder a ella en cuanto a recursos económicos; además, la oferta pública no alcanza a suplir la cantidad demandada y, sumado a esto, acceder a esta última para un bachiller que cuenta con una formación deficiente, poca orientación vocacional y bajos resultados en las pruebas de Estado Icfes resulta complejo (Ministerio de Educación Nacional, 2009, 2011).
Partiendo de lo expresado por Amartya Sen en su obra Desarrollo y libertad, el individuo cuenta con un espacio de deseo que, bajo condiciones restringidas, debe ser intervenido mediante acciones y saberes para convertirse en un espacio de realización, en el que se hallen capacidades de funcionamiento, posibilidades de autorelación, empoderamiento y ejercicio de la libertad de ser, hacer y estar (Comité Académico Organizador, 2013, p. 9). Desde esta visión, se busca paliar las problemáticas que vive la juventud en Santiago de Cali en cuanto a acceso a educación superior; es por ello que durante la administración del alcalde Jorge Iván Ospina (2008-2011) se implementó la política pública educativa para el plan de nivelación académica Talentos (PNAT), en convenio con la alcaldía de Cali y la Universidad del Valle, integrando esta última a su estrategia de extensión y proyección social, en la cual se planteaba como función el hecho de mejorar las competencias académicas para el ingreso a la educación superior y las condiciones de vida de los beneficiarios.

El convenio entre las instituciones tuvo como objetivo cumplir con múltiples procesos relacionados con la gestión académica, vinculando al proceso a profesores y monitores de la Universidad del Valle, el soporte tecnológico, la atención al proyecto - en cuanto a estructura física - y el apoyo en aspectos administrativos y de logística (Universidad del Valle, 2013, pp. 5, 6). En cuanto al aspecto financiero, se asignaron 4.150.000.000 millones para la creación del plan de nivelación académica Talentos. De estos se designaron 1.500.000.000 millones para ayudar con la movilidad de los estudiantes, otorgándoles a cada uno el valor de 100.000 pesos mensuales, durante los diez meses que duró 
cada cohorte; a la vez, 1000.000.000 millones se vieron representados en infraestructura y servicios (Vicerretoría Académica de la Universidad del Valle, 2012, citado por García Viera, 2013, p. 25).

La población tratamiento, considerada como la población por intervenir en la literatura del análisis de las políticas públicas, estuvo conformada en total por 4500 jóvenes durante las tres cohortes, teniendo cada una de las cohortes del plan 1500 estudiantes, los cuales debían ser bachilleres, menores de veintitrés años y pertenecer al estrato socioeconómico uno, dos y tres (este último específicamente en la tercera cohorte), lo cual se corroboró vía factura de los servicios públicos, y debían haber presentado las pruebas de Estado Icfes. La escogencia de los beneficiarios de la política pública se dio mediante un procedimiento similar al que se escogen o se seleccionan los estudiantes de la Universidad del Valle, teniendo como criterio de calificación las pruebas de Estado Icfes.

Esta política es la más holística al retomar el problema del abandono escolar a nivel superior en adolescentes con necesidades básicas insatisfechas, ya que en el fondo buscó paliar los factores que excluyen a los jóvenes de sectores deprimidos de la ciudad, pretendiendo incidir positivamente en el acceso a la educación y en el índice de violencia, (tratando la paz negativa); apostándole así a la temática de la educación para la paz y basándose en la construcción de un proyecto de vida en el ámbito académico y de la educación superior. Para ello su propuesta pedagógica estuvo consolidada sobre tres ejes: académico, con el que se planteó la nivelación en asignaturas; social, buscando la construcción de ciudadanos con capacidad crítica y puedan liderar procesos en pro de su comunidad; y cultural, que pretendió construir el proyecto académico paralelo al proyecto de vida de los alumnos (Vicerretoría Académica de la Universidad del Valle, 2012, citado por García Viera, 2013, p. 12).

La meta institucional fue mejorar la formación en cuanto a conocimientos en áreas básicas y de inclusión en la vida universitaria, para así proporcionar a la población tratamiento no solo la posibilidad de acceder a la educación superior, sino también a un subsidio (transporte), que de conjunto pretendían disminuir las desventajas ocasionadas respecto a calidad académica, capital humano, social y cultural, y a empoderamiento de la población; pues se pretendió formar ciudadanos que lideraran procesos de transformación en la sociedad, aprendiendo del reconocimiento como sujetos de derechos y la aceptación entre ellos (Universidad del Valle, 2013, p. 18).

Ahora bien, para la formulación e implementación del plan de nivelación académica Talentos se tuvieron en cuenta los factores relevantes en la producción educativa, como lo son las cuatro variables planteadas por Lassibille y Navarro (2004): entorno familiar y estatus socioeconómico, características de los entes educativos, entorno del aula de clases, y capital humano y características personales (Burbano Espinosa, 2012, p. 18).

Para impactar en estas variables se trabajaron las falencias que en la educación media y media-técnica se originaron, debido a que se descuidan en ella diversas áreas, entre estas las ciencias sociales, y que además la ampliación de cobertura privilegia actividades extracurriculares que llevan a descuidar los contenidos mínimos (Perafán Cabrera, 
s.f., citado por Martínez, s.f., p. 10). Por ello se desarrollaron módulos con el fin de nivelar áreas como matemáticas, lenguaje, física, biología, química, sociales, geografía, historia, filosofía y cultura ciudadana. A la vez se dictaron talleres de preparación para la vida universitaria, sobre emprendimiento empresarial, arte, conservación del patrimonio ambiental urbano, de gestión deportiva y formulación de proyectos (Universidad del Valle, 15 de septiembre de 2010); aspecto que mejoró la formación académica y la comprensión, al incrementar la postura crítica en situaciones de la vida pública y sobre la realidad que habitan (Martínez. p. 16).

Con el fin de incidir en el entorno de vida o en el escenario comunitario de los alumnos, se ejecutó el componente en cultura ciudadana, en el que se construyeron espacios para promover los valores de integración social y de respeto por el otro, motivando el intercambio de conocimientos y experiencias de aprendizaje que sirvieran de base y como motor de educación para la paz; estimulando la reflexión sobre el proyecto de vida, la vinculación a la vida universitaria y la consolidación de una nueva cultura educativa, la cual respondiera a las demandas sociales e institucionales con respecto al plan de Gobierno municipal y al plan sectorial educativo.

Para que los estudiantes impactaran los contextos sociales, se realizaron módulos sobre servicio social, así ellos participaron en actividades cívicas, ecológicas, de cuidado al medio ambiente y de alfabetización; de esta forma, actuaron en diversos escenarios, fomentaron el respeto por la vida y las normas de cultura ciudadana, y construyeron pedagógicamente a la ciudad (Contraloría General de Santiago de Cali, 2011, p. 13).
El plan Talentos como impulsor de educación para la paz tiende a plantear los principios de esta, e impulsa así el cultivo de los valores, tales como la justicia, la cooperación, la solidaridad, el desarrollo de la autonomía y la toma de decisiones; el educar en el diálogo y la argumentación racional, en la tolerancia y diversidad, y en la resolución de conflictos; en el desarrollo del pensamiento crítico y en el aprender a vivir con los demás; cuestionando a la vez los valores antitéticos a la cultura de la paz como la discriminación, intolerancia, etnocentrismo, insolidaridad, inconformismo, entre otros (Zurbano Díaz de Cerio, 1999, pp. 21-22).

Al seguir la metodología de la educación para la paz, el programa trabajó temáticas concernientes a la cultura democrática y la formación ciudadana, la convivencia y la paz, la participación democrática y la responsabilidad, la pluralidad, la identidad y valoración de las diferencias, la división de poderes, la democracia como tal y la promoción y defensa de los derechos humanos. Mediante esto cumple con las tres actitudes básicas para este tipo de educación: el pluralismo, que valora tanto la igualdad como la diversidad; la tolerancia, hacia todo lo que contradice las ideas y valores individuales, y el diálogo, como principal instrumento en el momento de resolver conflictos (Zurbano Díaz de Cerio, 1999, p. 66).

Cumpliendo con lo planteado en la educación para la paz en cuanto a los tres niveles por trabajar en la población beneficiaria, se encontró que el cognitivo expresa sobre el aprender conocimientos y principios teóricos relacionados con la educación para la convivencia pacífica; el afectivo se refiere al hecho de fomentar actitudes, y el conductal señala 
que las conductas responden a la exigencia en la vida diaria (Zurbano, 1999, pp. 152-154). Se origina, partiendo del proyecto "Tejer Desde Adentro: La Universidad Imaginada", la Secuencia de Actividad Recreativa Intensiva (Sari), que tuvo como objetivo incidir en los alumnos en cuanto a la orientación vocacional y en la creación de aptitudes para el ejercicio de la ciudadanía (Mesa y Manzano, 2010, citados por García Viera, 2013, p 13).

De esta manera, se trabajaron en este espacio una serie de secciones que tocaron temáticas como historias de vida, toma de decisiones, cuestiones de reconocimiento y como actividad la creación de un mini-carnaval. Con esto se quiso identificar el contexto social donde residían los alumnos, trabajar el reconocimiento hacia sí mismo y los demás, y expresar los imaginarios y expectativas entorno al ingreso a la educación superior y la vida universitaria (García Viera, 2013, pp. 17-19).

\section{Resultados del PLAN DE NIVELACIÓN ACADÉMICA TALENTOS}

El plan Talentos tuvo una duración de tres cohortes y cada una trabajó en promedio alrededor de 32 semanas (un año académico universitario); mediante estas se consolidó como una buena opción para incidir en la nivelación académica, en comparación con la iniciativa privada, ya que sus costos por hora de nivelación fueron menores y su intensidad horaria fue tres veces superior (Burbano Espinosa, 2012, p. 37).

La población que ingresó al plan de nivelación académica Talentos, considerada como la población tratamiento en la literatura del análisis de las políticas públicas, estuvo conformada por 4500 admitidos, de los cuales
3276 finalizaron el programa; y de estos 1619 lograron ingresar a la educación superior; a esta última accedió el $36 \%$ de la población total. Uno de los logros relevantes del plan Talentos fue trabajar en la población tratamiento con jóvenes cuyas circunstancias personales y características de su entorno necesitan intervención para el no incremento en el índice de violencia y las expresiones agresivas; todo lo anterior contribuye a una verdadera educación para la paz.

Con respecto a las cohortes, la primera cohorte estuvo constituida por 1187 participantes, de estos, 616 accedieron (51,86\%) y de los cuales 476 ingresaron a la Universidad del Valle. En cuanto a la segunda cohorte, ingresaron 592 de 1043 alumnos $(56,75 \%)$, de los cuales 533 accedieron a la Universidad del Valle; y la tercera cohorte presentó un total de 1047 participantes de los cuales ingresaron a la educación superior 411 (39,25\%), de los cuales 388 accedieron a la Universidad del Valle.

Partiendo de lo planteado por Cunha y Heckman (2007), sobre el hecho de que la mitad de la desigualdad en los ingresos de la vida se debe a factores determinados a los 18 años de edad, se encuentra que en la población impactada por el programa hay un gran porcentaje de alumnos entre los 18 y 23 años. De esta manera, la primera promoción contó con el 91,19\% de población entre esta edad; la segunda tuvo un promedio de $92,39 \%$; y la tercera estuvo conformada por un 91,38\%. Se buscó así paliar la desigualdad dada a dicha edad.

La incidencia en el contexto social fue notoria, ya que el plan estuvo conformado por jóvenes de los estratos socioeconómicos uno, dos y tres; en especial hubo población 
de la zona oriental de la ciudad, específicamente de las comunas $6,7,13,14,15,16$ y 21, población considerada como la más excluida, con violaciones de derechos humanos, con mayores necesidades insatisfechas, con altos indicadores de violencia en sus entornos, y en muchas ocasiones sin oportunidades laborales y de formación académica.

Durante las tres cohortes hubo mayor porcentaje de mujeres, las cuales correspondieron en la primera cohorte al 66,92\%; en la segunda, al $57,74 \%$; y en la tercera, al 59,87\%. En cuanto a la situación socioeconómica del alumnado, en la primera cohorte se contó con un porcentaje del $44,89 \%$ en estrato uno, y el 55,11 \% pertenecía al estrato dos; la segunda cohorte se conformó por un $42,88 \%$ por estudiantes pertenecientes al estrato uno, siendo el 57,12\% del estrato dos. Para la tercera cohorte en estrato uno fue de $33,72 \%$; en el estrato dos hubo un 54,02\%; y para esta ocasión se conto con alumnos cuya situación socioeconómica correspondía al estrato tres, correspondientes al 12,26 \% de la población.
Con respecto al impacto social, cabe destacar que el plan de nivelación académica Talentos al tener un gran porcentaje de población de la zona oriental de la ciudad - tratando de esta manera una población realmente necesitada de políticas de inclusión social-, cumple con incidir en la situación socialmente problemática que vive la ciudad, ya que atiende la situación correcta (acceso a la educación superior en jóvenes), sin generar la problemática y la demanda desde la institucionalidad, sino que se constituye como respuesta desde la problemática y la población como tal. De esta manera, durante las tres cohortes se observó que más del $50 \%$ de la población pertenecía al sector oriental de la ciudad; así, durante la primera cohorte la población de este sector correspondió a un 63,11\%; en la segunda, a un 65,53\%; y en la tercera, al 58,52\% de la población total.

Ahora bien, en cuanto al impacto de la política pública sobre el mejoramiento de las competencias en la población tratamiento, se presenta lo siguiente:

Figura 1. Talentos primera cohorte

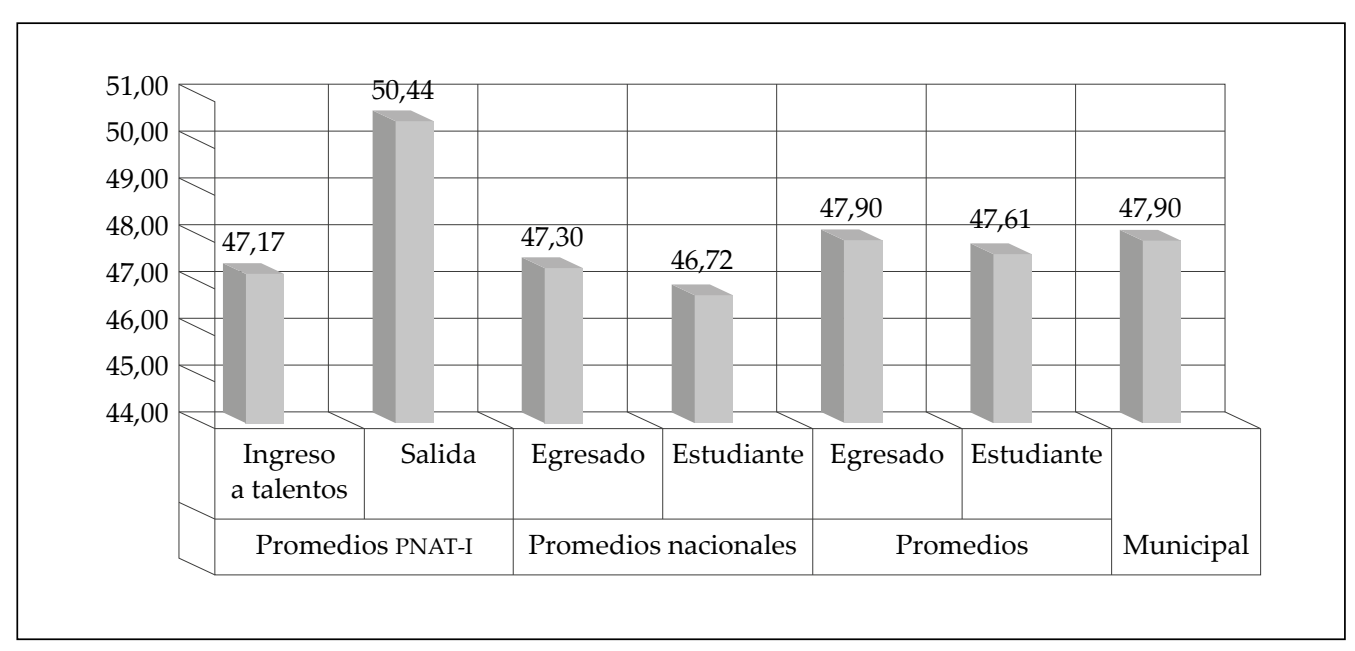

Fuente: elaboración propia, datos tomados a partir de Extensión

y Educación Continua, Universidad del Valle (2013) 
Figura 2. Talentos segunda cohorte

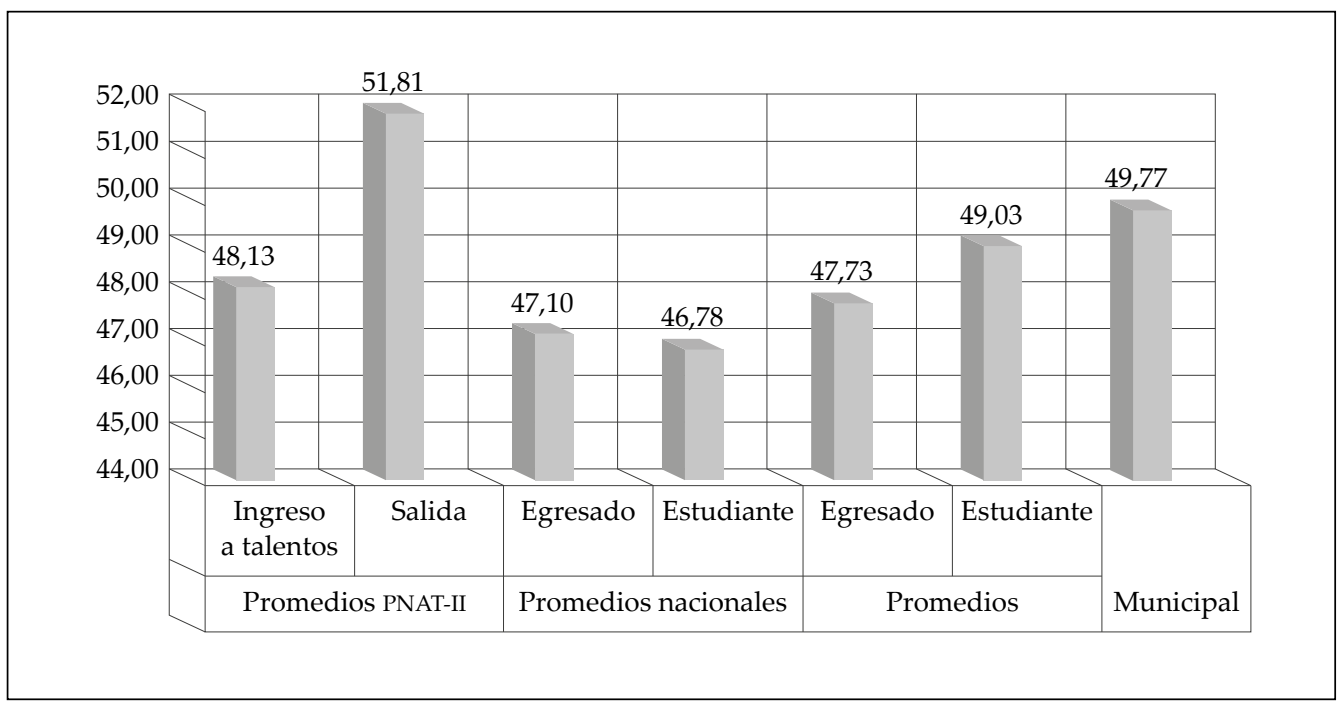

Fuente: elaboración propia, datos tomados a partir de Extensión y Educación Continua, Universidad del Valle (2013)

Figura 3. Talentos tercera cohorte

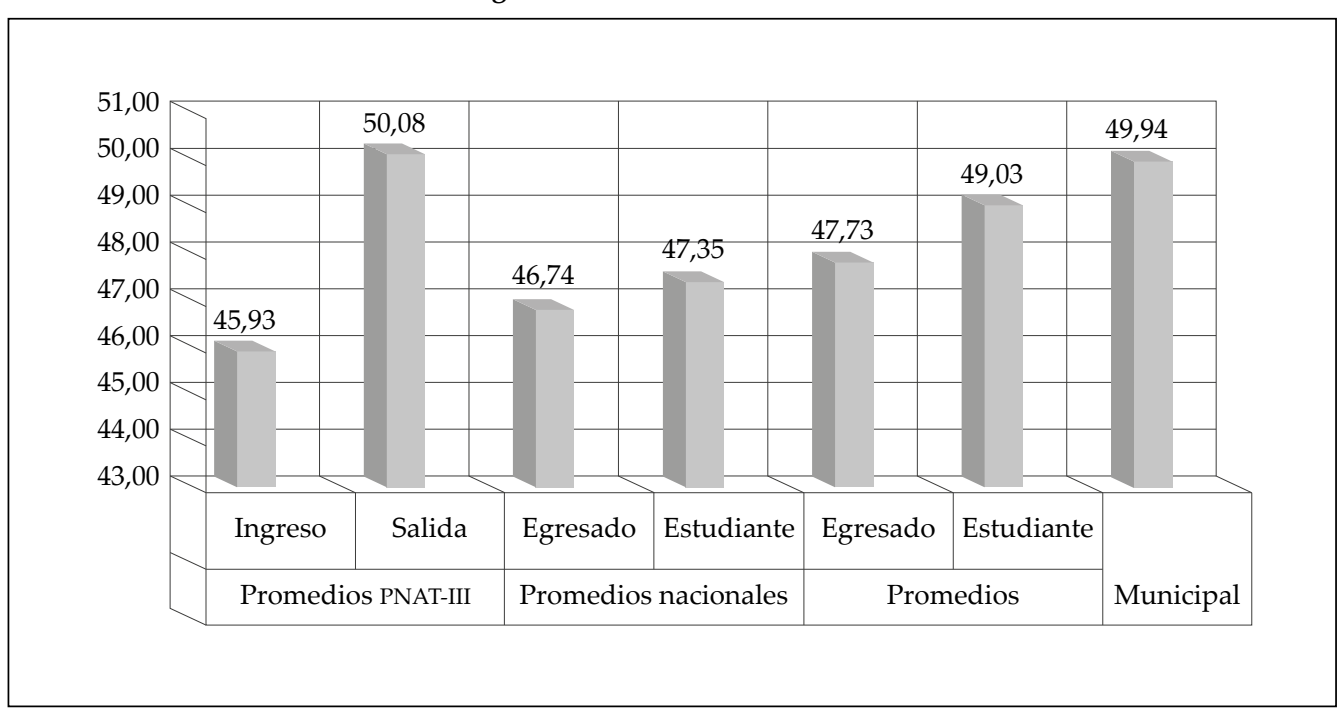

Fuente: elaboración propia, datos tomados a partir de Extensión y Educación Continua, Universidad del Valle (2013) 
En cuanto a los resultados en las pruebas de estado Icfes, la población tratamiento posterior a su formación en el plan Talentos realizó nuevamente la prueba Saber11 y sus resultados mejoraron alrededor de cuatro puntos porcentuales por encima de la media, correspondiendo esta al $47 \%$; en las cohortes se tienen cifras al ingresar de $47,17 \sim 1$ \% (1), 48,13\% (2) y 45,93 \% (3); y al salir se obtuvieron cifras de 50,44\% (1), 51,81 \% (2), y 50,08 \% (3) (Comité Académico Organizador, 2013, pp. 22-24) ${ }^{1}$. Por lo tanto, se evidencia la efectividad del programa del plan de nivelación académica
Talentos, dado que mejora los puntajes de los participantes, es decir, se elevan los niveles de formación académica por el bajo capital humano con el que ingresan. Claro está, no hay que pasar por alto que la tendencia de los promedios municipal, departamental y nacional de las pruebas Saber11 durante 2009, 2010 y 2011 se encuentra en alrededor del $47 \%$, lo cual muestra que los estudiantes que ingresan al programa representan fielmente a la población control, conformada por los estudiantes que participan de la política.

Figura 4. Impacto del plan talentos

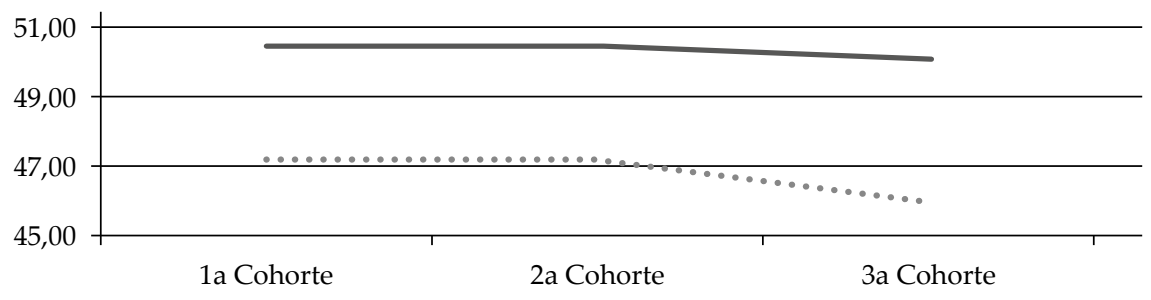

-... Puntaje de ingreso a talentos

— Puntaje ex post de talentos

Fuente: elaboración propia, datos tomados a partir de Extensión y Educación Continua, Universidad del Valle (2013)

1 Se realizó este ejercicio evaluativo de política pública, con base en la información generada por Julieta Muriel (coordinadora administrativa del proyecto). 


\section{Con respecto al desempeño de los estudiantes}

en las pruebas de núcleo común del Icfes se encuentra lo siguiente:

Figura 5. Comparación resultados pruebas Saber11 vs. PNAT I

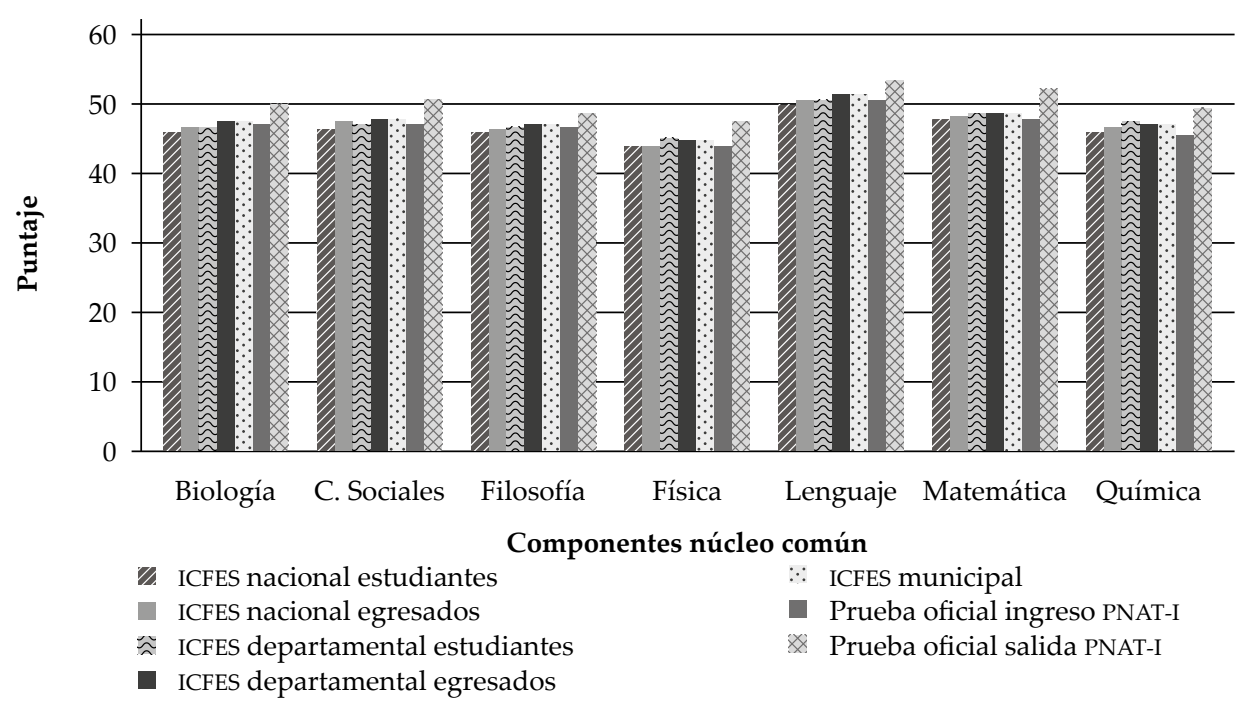

Fuente: elaboración propia, datos tomados a partir de Extensión y Educación Continua, Universidad del Valle (2013)

Figura 6. Comparación resultados pruebas Saber11 vs. PNAT II

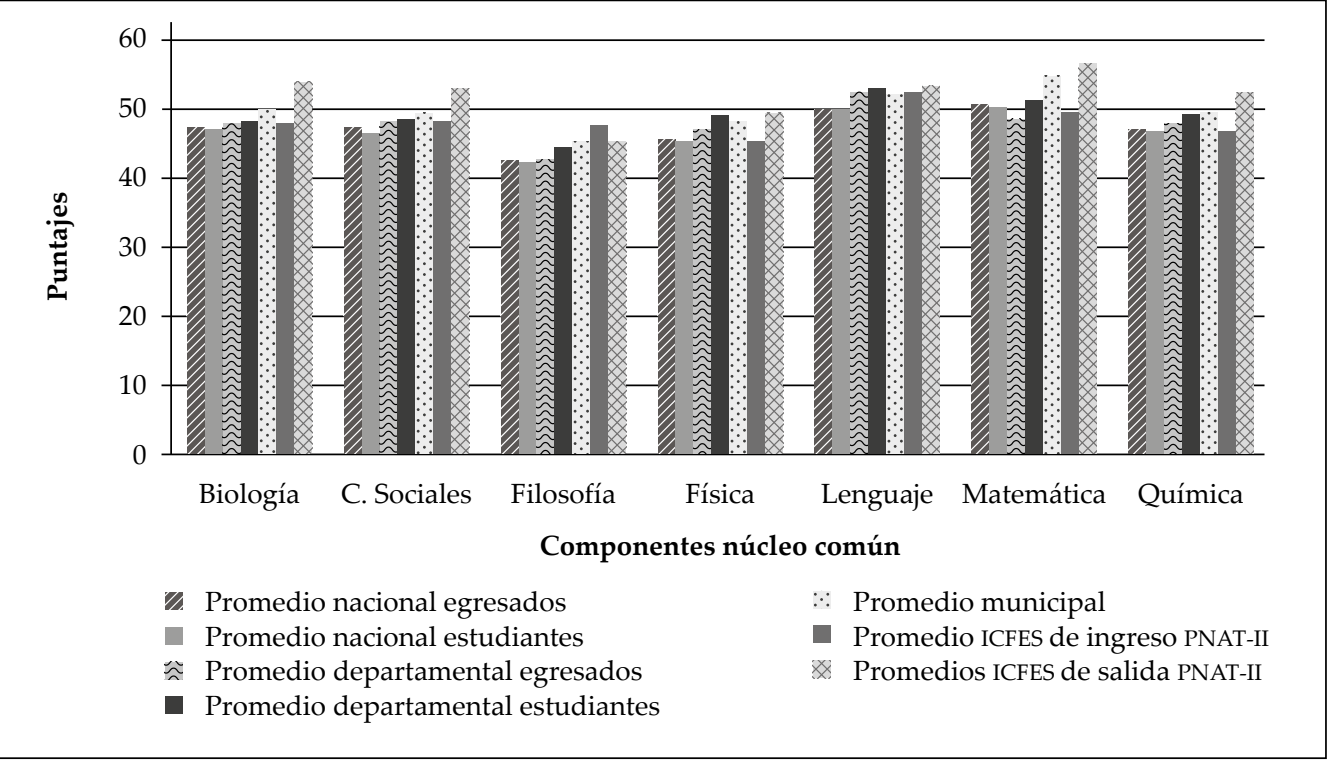

Fuente: elaboración propia, datos tomados a partir de Extensión y Educación Continua,

Universidad del Valle (2013) 
Figura 7. Comparación resultados pruebas Saber11 vs. PNAT III

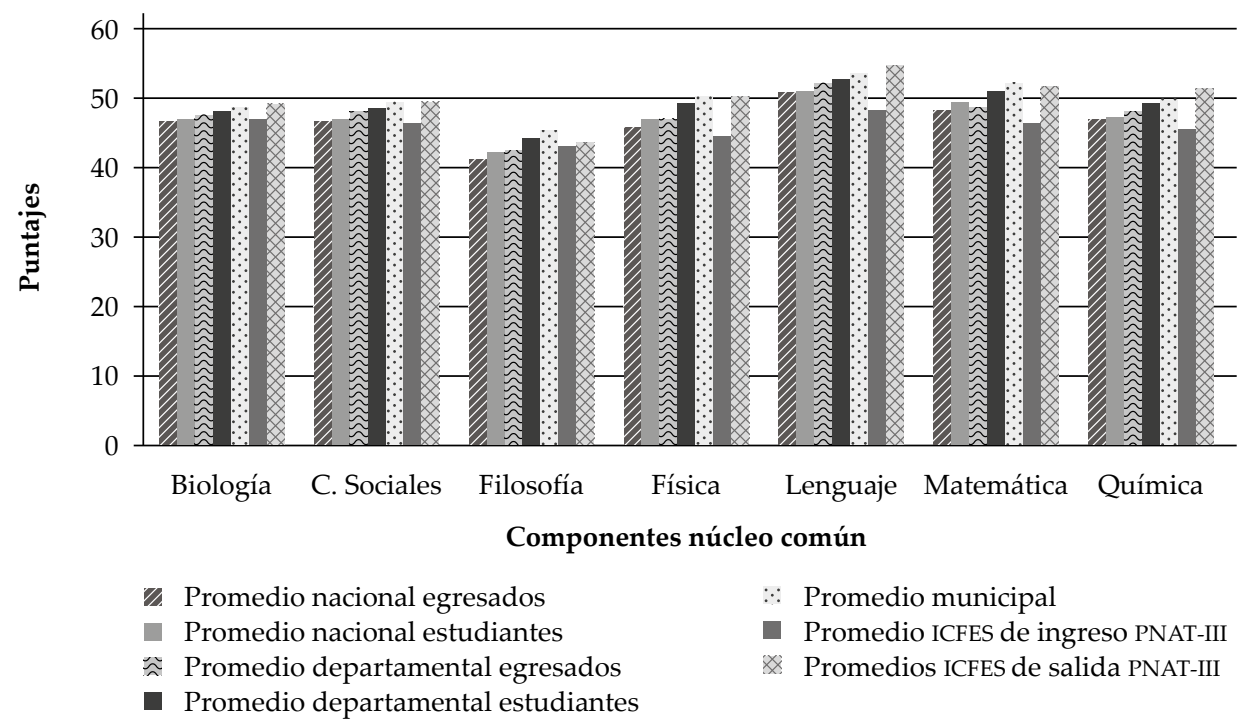

Componentes núcleo común

V. Promedio nacional egresados

- Promedio nacional estudiantes

$\approx$ Promedio departamental egresados

- Promedio departamental estudiantes

$\therefore \quad$ Promedio municipal

- Promedio ICFES de ingreso PNAT-III

Promedios ICFES de salida PNAT-III

Fuente: elaboración propia, datos tomados a partir de Extensión

y Educación Continua, Universidad del Valle (2013)

En el núcleo común de las pruebas de Estado Icfes se observa una mejoría en cuanto al promedio, dado al ingreso y a la salida del programa, y al promedio de salida y el municipal, en cada área. De esta manera, los estudiantes en el área de biología ingresaron en la primera cohorte con un promedio de 47,21, el cual mejoró a 50,08 al salir, siendo superior al municipal que corresponde al 47,34 . La segunda cohorte en la misma área ingresó con un promedio de 47,63 y al finalizar aumentó al 53,60 siendo mayor al municipal que fue de 49,87; y la tercera cohorte al iniciar tenía un promedio de 46,90; egresó con uno de 49,26; estando este por encima del municipal cuyo promedio era 48,74.

En el área de ciencias sociales, la primera promoción conto con un puntaje de 47,14, el cual mejoro a 50,82 por encima del municipal que estaba en 48,26 ; la segunda cohorte tuvo al inicio un porcentaje de 48,15 que pasó a ser un 52,88, siendo superior al municipal que era 49,41. En cuanto a la tercera promoción, esta ingresó con un promedio de 46,58 que pasó a estar en 49,41, quedando cerca al municipal que estaba en 49,37. En lenguaje se ingresó con un promedio de 50,82, el cual aumentó a 53,5 superior al municipal que estaba en 51,62. La siguiente cohorte tuvo al iniciar un puntaje de 52,28, el cual aumentó casi un punto a 53,10, estando cerca del promedio municipal que era 51,94; y la última promoción ingresó con un porcentaje de 48,45 , aumentando a 54,70, casi un punto por encima del promedio municipal que se encontraba en 53,61.

El área de filosofía tuvo en la primera promoción un promedio de 46,87, el cual mejoró a 48,95 , por encima del municipal que estaba en 47,09; la siguiente cohorte inició con un 
porcentaje de 47,46, que disminuyó luego a 45,28 , siendo aún superior al municipal que era 45,23. Para la tercera cohorte se contó con un promedio inicial de 43,09, que posteriormente pasó a estar en 43,80; quedando cerca al municipal que estaba en 45,34 . Ahora bien, en física se contó en la primera cohorte con un promedio de 44,16, que pasó a ser 47,52, por encima del municipal que era 44,83 ; la segunda cohorte inició con un puntaje de 45,19 , el cual aumentó a 49,44, por encima del municipal que correspondía a 47,98; y la última promoción inició con un promedio de 44,47 , la cual luego aumentó a 50,38, quedando cerca al municipal que estaba en 50,33.

En cuanto a matemáticas, las cohortes iniciaron con promedios de 48,16 (1), 49,53 (2) y 46,35 (3), los cuales se modificaron a 52,44 (1), 56,19 (2) y 52,63 (3); y quedaron por encima de los promedios municipales que fueron 48,97 (1), 54,46 (2) y 52,29 (3). El área de química tuvo en la primera cohorte un porcentaje de 45,86 el cual aumentó a 49,76 , siendo superior al nivel municipal que era 47,18; la segunda promoción inició con un puntaje de 46,68 , el cual aumentó alrededor de seis puntos a 52,20, quedando por encima del promedio municipal que estaba en 49,49. La última cohorte ingresó con un porcentaje de 45,70 que pasó a ser 51,37, estando superior al municipal que fue 49,87. Así, en todas las áreas del núcleo común de las pruebas de Estado Icfes se aumentaron los promedios, hecho que demuestra una mejoría en el conocimiento, y por ende en el capital humano de la población tratamiento.

Para finalizar, desde el punto de vista financiero, hay que tener en cuenta que con menores recursos (ingresos) (Burbano
Espinosa, 2012, p. 37) se logró mucho más de lo que realmente cuesta desarrollar un programa de esta magnitud; por ejemplo, si se desarrollase este programa por parte del sector privado sería mucho más costoso, sin garantía de una mejora en los niveles de conocimiento y sin subsidio. Como se logra vislumbrar, el plan talentos genera un impacto mayor en proporción a los costos económicos y sociales del proyecto, $\mathrm{y}$ en relación con sus resultados (como meta o logro deseado de una política pública, que es la teoría del cambio social y la creación de valor público). Esto implica que sea un reto el educar a los participantes durante ocho meses para mejorar en cuatro puntos porcentuales, puntajes inferiores al promedio municipal y a la vez mejorar doce años de educación heterogénea de mala calidad. Como se puede notar, el programa impulsado desde la administración de Jorge Iván Ospina Gómez (2008-2011) y desarrollado por la Universidad del Valle fue todo un éxito, y se logró incluso la “Exaltación al Mérito Educativo y Pedagógico 2012". (Universidad del Valle, 19 de abril de 2012).

\section{Referencias}

Alcaldía de Santiago de Cali (2008). Plan de desarrollo de Santiago de Cali 2008-2011 "Para vivir dignamente". Resumen ejecutivo. Santiago de Cali.

Burbano Espinosa, C. (2012). Una Aproximación a los determinantes del éxito del plan de nivelación académica Talentos (Trabajo de grado). Universidad del Valle, Santiago de Cali.

Castrillón Córdoba, M. (2013). Incidencia de la desigualdad socioeconómica sobre la inversión de capital humano: un estudio para 
Cali en el 2005 (Trabajo de grado). Universidad del Valle, Santiago de Cali.

Comité Académico Organizador (agosto de 2013). Plan de nivelación académica Talentos. Propuesta académica cohorte IV. Santiago de Cali: Universidad del Valle.

Contraloría General de Santiago de Cali (9 de marzo de 2011). Seguimiento al Programa Plan Talentos - Municipio de Santiago de Cali-Secretaria de Educación Municipal, Santiago de Cali.

Cunha, F. y Heckman, J. J (2007). Investing in Our Young People (unpublished manuscript). Chicago: University of Chicago.

Cunha, F. y Heckman, J. (2008). A New Framework for the Analysis of Inequality. Macroeconomic Dynamics, 12(52), 315-354.

García Viera, S. I. (2013), Analisis del proyecto metodologico de una sesión de trabajo orientada por la metodología de intervención SARI (Trabajo de grado). Universidad del Valle, Santiago de Cali.

Heckman, J. J. (2000). Policies to Foster Human Capital. Research in Economics, 54(1), 3-56.

Leyva, S. y Cardenas, A. (2002). Economía de la educación, capital humano y rendimiento educativo. Análisis Económico, XVII (036), 79-106.

Ministerio de Educación Nacional (2008). Informe de Gestión 2002-2010. Bogotá: Ministerio de Educación Nacional.
Schultz, T. W. (1968). Human Capital. New York: McMillan Company.

Universidad del Valle (marzo de 2013). Plan de nivelación académica Talentos [PNAT], Informe Final Plan, Santiago de Cali.

Ídem (15 de septiembre de 2010). El éxito del plan talentos. Campus, un periódico quincenal, 96. Recuperado el 2 de febrero de 2014, de

http://campus.univalle.edu.co/2010/septiembre/edicion96/talentos.php

Ídem (19 de abril 19 de 2012). Reconocimiento para el plan de nivelación académica Talentos. Recuperado el 3 de febrero de 2014, de http://comunicaciones.univalle.edu.co/InformesPrensa/2012/abril/OC-062-2012. html

Vergara Varela, R. (marzo de 2011). Análisis de la Política Pública de los Desayunos Escolares (2010). Una Aproximación Multidisciplinar. Santiago de Cali: Programa de Estudios Políticos y Resolución de Conflictos, Instituto de Educación y Pedagogía [IEP], Universidad del Valle. Zurbano Díaz de Cerio, J. L. (1999). Bases de una educación para la paz y la convivencia. Pamplona: Gobierno de Navarra., Departamento de Educación y Cultura. 